\title{
Electrochemical Growth of Three-Dimensional Nanostripe Architecture of Antimony on $\mathrm{Cu}(100)$
}

Ji-Hong Wu ${ }^{1}$, Jia-Wei Yan ${ }^{1}$, Zhao-Xiong Xie ${ }^{1}$, Qi-Kun Xue ${ }^{2}$, Bing-Wei Mao ${ }^{1 *}$

\section{Supporting information}

Figure 1S. In-situ STM images before and after the supply of more Sb(III) containing solution to STM cell for further growth.

Figure 2S. In-situ STM image showing Sb nanostripes formed in high acidic media. 


\section{Figure 1S}

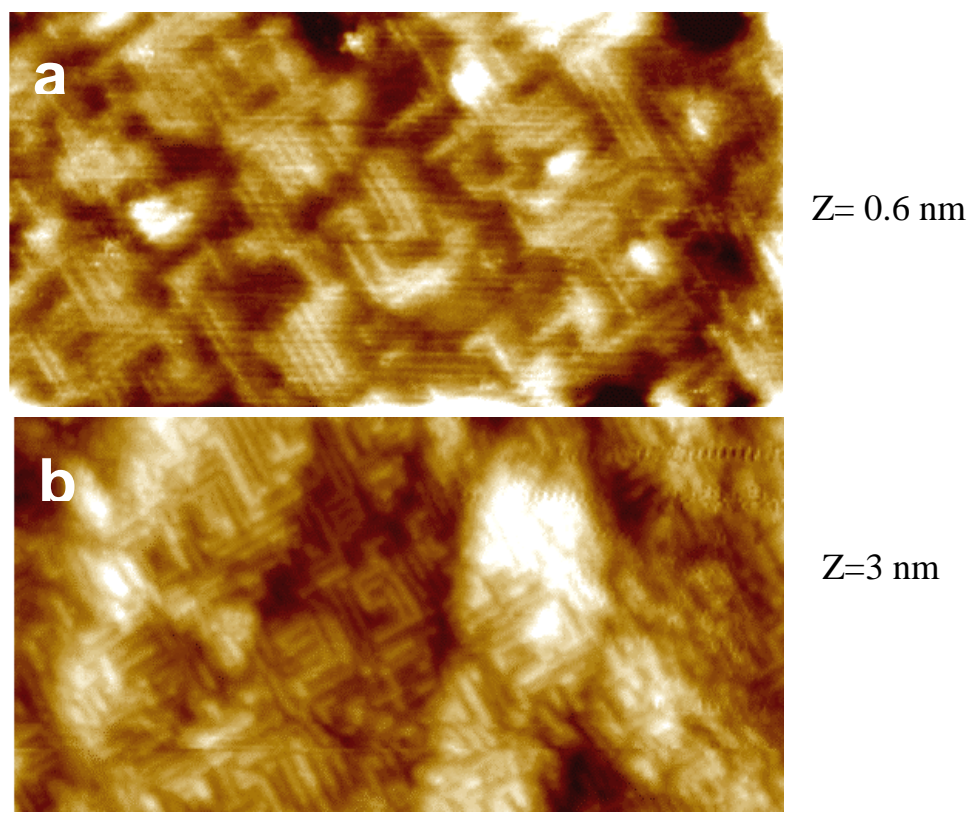

Figure 1S STM images before (a) and after (b) the supply of more concentrated $\mathrm{Sb}$ (III) containing solution to STM cell for further growth. The images were recorded in different areas, but nanostripes kept the same orientations and run exclusively in two perpendicular directions. The apparent bright protrusion in the upper right region of frame $b$ also contains orientated nanostripes, which can be viewed if with proper contrast. The results imply that a regular straw-mat type of stacking feature preserved throughout the growth process. Scan size: $75 \mathrm{~nm} \times 37.5 \mathrm{~nm}$. 


\section{Figure 2S}

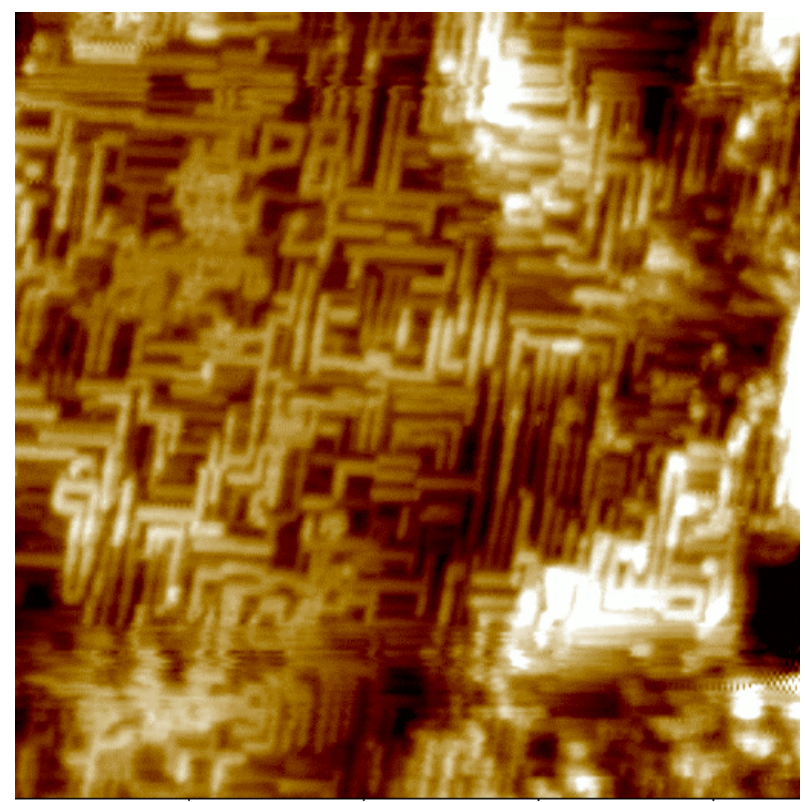

Figure $2 S$. In-situ STM image showing Sb nanostripe film formed at. - $0.35 \mathrm{~V}$ in $0.5 \mathrm{M} \mathrm{H}_{2} \mathrm{SO}_{4}+40 \mu \mathrm{M} \mathrm{Sb}$ (III ). In this high acidity solution, the length of the nanostripes is increased up to $\sim 20 \mathrm{~nm}$, but the order of the nanostripes is somewhat reduced. Scan size: $90 \mathrm{~nm} \times 90 \mathrm{~nm}$.

The growth of nanostripe was observed in the $\mathrm{Sb}(\mathrm{III})$ concentration up to $\sim 100 \mu \mathrm{M}$ in sulfuric or perchlorate acid solution of $10 \mathrm{mM} \sim 0.5 \mathrm{M}$ at overpotentials larger than $100 \mathrm{mV}$. Among these growth patterns, only the length and the ordering of the nanostripes were slightly influenced with varying conditions. 